\title{
Percepções sobre a plataforma Google Classroom como ferramenta de apoio ao ensino-aprendizagem durante a pandemia da COVID-19
}

Insights into the Google Classroom platform as a tool to support teaching-learning during the COVID-19 pandemic

Percepciones sobre la plataforma Google Classroom como herramienta de apoyo a la enseñanzaaprendizaje durante la pandemia de COVID-19

Bianca Maria Beserra Costa ORCID: https://orcid.org/0000-0002-8132-9881 Universidade de Pernambuco, Brasil E-mail: bianca.bcosta@upe.br Jorge Miguel Albrecht Ribeiro Filho ORCID: https://orcid.org/0000-0002-3772-1851

Faculdade de Odontologia do Recife, Brasil

E-mail: Jorge_albrechtfilho@ hotmail.com

Paulo Maurício Reis de Melo Júnior ORCID: https://orcid.org/0000-0001-9926-5348 Universidade de Pernambuco, Brasil E-mail: Paulo.reis@upe.br

Sandra Maria Alves Sayão Maia ORCID: https://orcid.org/0000-0003-3172-2332

Universidade de Pernambuco, Brasil

E-mail: sandrinhasayao@hotmail.com

Luciano Barreto Silva

ORCID: https://orcid.org/0000-0002-1508-4812

Faculdade de Odontologia do Recife, Brasil E-mail: Luciano.barreto@for.edu.br

Natália Gomes de Oliveira

ORCID: https://orcid.org/0000-0001-6937-1537

Universidade de Pernambuco, Brasil

E-mail: nataliagomes04@hotmail.com

Luís Felipe Espíndola-Castro

ORCID: https://orcid.org/0000-0002-1923-8057

Universidade de Pernambuco, Brasil

E-mail: lipe_espindola@hotmail.com

Marina da Cunha Isaltino

ORCID: https://orcid.org/0000-0002-8991-2721

Faculdade de Odontologia do Recife, Brasil

E-mail: marinaisaltino93@gmail.com

Pâmella Recco Álvares

ORCID: https://orcid.org/0000-0003-3396-9339

Universidade de Pernambuco, Brasil

E-mail: pamella.recco@upe.br

Juliana Syndia Silva Santos Sousa

ORCID: https://orcid.org/0000-0001-9572-0593

Faculdade de Odontologia do Recife, Brasil

E-mail: julianasyndia@gmail.com

Letícia Lopes de Arruda Diniz

ORCID: https://orcid.org/0000-0003-0017-7263

Faculdade de Odontologia do Recife, Brasil E-mail: leticialopesdea@gmail.com

Diogo Cabral de Melo Filho

ORCID: https://orcid.org/0000-0003-4239-109X

Universidade de Pernambuco, Brasil

E-mail: diogo.odonto1@gmail.com

Iasmin Cirino da Silva

ORCID: https://orcid.org/0000-0001-5939-4524

Universidade de Pernambuco, Brasil

E-mail: iasmincirino@gmail.com 


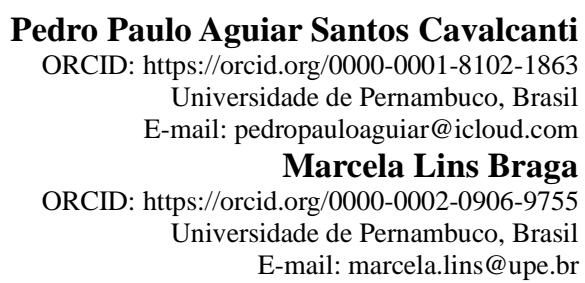

\begin{abstract}
Resumo
Neste trabalho, objetivou-se avaliar a percepção dos estudantes da Faculdade de Odontologia de Pernambuco sobre o uso do aplicativo Google Classroom, como ferramenta de apoio ao ensino-aprendizagem durante o período suplementar letivo trans pandêmico. A amostra foi composta por 100 alunos do primeiro ao décimo período. O formulário on-line foi constituído por 19 itens de múltipla escolha e os resultados foram tabulados pela própria plataforma em planilhas (via Google Sheets), sendo visualizados em gráficos. Para tanto, os estudantes tiveram acesso ao convite da pesquisa através de um link do formulário on-line, que foi encaminhado pelo pesquisador por meio de mensagens aos grupos de WhatsApp das turmas de estudantes. Os dados foram analisados mediante estatística descritiva (média e desvio padrão, valor máximo e mínimo), considerando como nível de significância p-valor $\leq 0,05$ para um intervalo de confiança de $95 \%$. Os estudantes da Faculdade de Odontologia de Pernambuco aprovaram a usabilidade e interação do aplicativo Google Classroom como ferramenta virtual de ensino-aprendizagem, mostrandose adequado e eficaz, quando utilizado nas disciplinas cursadas no período letivo suplementar, durante a pandemia da COVID-19.
\end{abstract}

Palavras-chave: Pandemia; Google Classroom; Odontologia; Ensino.

\begin{abstract}
In this paper, we aimed to evaluate the perception of students at the Pernambuco School of Dentistry about the use of the Google Classroom application as a tool to support teaching-learning during the trans pandemic supplementary school period. The sample was composed of 100 students from the first to the tenth period. The online form consisted of 19 multiple choice items and the results were tabulated by the platform itself in spreadsheets (via Google Sheets), and visualized in graphs. For this, the students had access to the survey invitation through a link on the online form, which was forwarded by the researcher through messages to the WhatsApp groups of the students' classes. Data were analyzed using descriptive statistics (mean and standard deviation, maximum and minimum value), considering as level of significance $p$-value $\leq 0.05$ for a confidence interval of $95 \%$. The students from the Pernambuco School of Dentistry approved the usability and interaction of the Google Classroom application as a virtual teaching-learning tool, proving to be adequate and effective when used in the subjects studied in the supplementary school period during the COVID-19 pandemic.
\end{abstract}

Keywords: Pandemic; Google Classroom; Odontology; Teaching.

\title{
Resumen
}

Este estudio tuvo como objetivo evaluar la percepción de los alumnos de la Facultad de Odontología de Pernambuco sobre el uso de la aplicación Google Classroom, como herramienta de apoyo a la enseñanza-aprendizaje durante el período escolar complementario transpandémico. La muestra estaba compuesta por 100 estudiantes del primer al décimo periodo. El formulario online constaba de 19 ítems de opción múltiple y los resultados fueron tabulados por la propia plataforma en hojas de cálculo (a través de Google Sheets), siendo visualizados en gráficos. Para ello, los alumnos tuvieron acceso a la invitación a la encuesta a través de un enlace en el formulario online, que fue remitido por el investigador a través de mensajes a los grupos de WhatsApp de las clases de los alumnos. Los datos se analizaron mediante estadísticas descriptivas (media y desviación estándar, valor máximo y mínimo), considerando como nivel de significación un valor $\mathrm{p} \leq 0,05$ para un intervalo de confianza del 95\%. Los alumnos de la Facultad de Odontología de Pernambuco aprobaron la usabilidad e interacción de la aplicación Google Classroom como herramienta virtual de enseñanza-aprendizaje, demostrando ser adecuada y eficaz cuando se utilizó en las asignaturas estudiadas en el período académico complementario durante la pandemia de COVID-19.

Palabras clave: Pandemia; Google Classroom; Odontología; Enseñanza.

\section{Introdução}

A relação direta entre estudo e tecnologia tem se mostrado necessária diante da inovação da abordagem pedagógica, exigindo mudanças no processo e na reflexão crítica sobre a interação professor, estudantes e seus pares. O incentivo às novas formas de ensino e aprendizagem têm sido propostos em todos os níveis de educação. O estudante precisa exercer 
ativamente o papel de protagonista, reconhecendo o professor como mediador entre o conhecimento elaborado e as dúvidas surgidas, ao invés de unicamente, apresentações sobre o conteúdo da disciplina (Silva, 2018).

Caminhos inovadores para a capacitação e formação dos profissionais estão sendo adotados em formas diferenciadas de ensino-aprendizagem e de organização curricular, na perspectiva de interligar a teoria com a prática e o ensino com o serviço, além de desenvolver a capacidade reflexiva acerca de problemas reais, na formulação de ações originais e criativas capazes de modificar a realidade social (Marin et al., 2016).

A educação superior na área da saúde passa por profundas mudanças para acompanhar as correntes de pensamento que norteiam a formação do profissional do docente, por isso, de acordo com os pressupostos nas Diretrizes Curriculares Nacionais para os cursos de Odontologia, a utilização de novas estratégias e metodologias ativas é definida como prioridade, e devem estar descritas nos projetos pedagógicos dos cursos como estratégias de ensino-aprendizagem, havendo necessidade da realização de projetos de intervenção para o desenvolvimento de experiências educacionais (Melo Júnior et al., 2018).

As Tecnologias da Informação e Educação (TIC) estão cada vez mais presentes em nosso cotidiano. Na educação, ela se apresenta como um valioso recurso no auxílio ao processo de ensino-aprendizagem. Nessa conjuntura, os profissionais de educação possuem um papel muito importante neste cenário, na execução das respectivas tecnologias, dominando a técnica e o planejamento pedagógico minucioso. (Araújo, 2016)

No cenário tecnológico, destacam-se especialmente a utilização de aplicativos móveis (também conhecidos como apps - do inglês application). Os aplicativos são conhecidos como um conjunto de ferramentas programadas para realizar e executar tarefas e atividades específicas. Essas plataformas podem ser acessadas em qualquer tipo de dispositivo, possibilitando queda de barreiras de tempo e espaço (Barra et al., 2017).

Diante da situação atual do País, no enfrentamento à pandemia da COVID-19 e de acordo com as recomendações da Organização Mundial da Saúde (OMS) sobre adoção de medidas de distanciamento e isolamento social, como forma de diminuir a propagação do vírus, a Universidade de Pernambuco (UPE) autorizou em caráter exclusivamente emergencial e provisório, o ensino remoto e a adoção de um período letivo suplementar, no qual as atividades de ensino fossem realizadas por meio de ferramentas de tecnologias de informação e comunicação (Moodle, OpenREDU e Google Classroom), como soluções transitórias para os estudantes da graduação presencial, regularmente vinculados ao semestre 2020.1 (primeiro semestre de 2020).

O Google Classroom ou Google Sala de Aula é um software para ambientes educacionais, componente da suíte de aplicativos Google for Education, desenvolvidos para o fomento e utilização na educação, juntamente à ferramenta de e-mail (GMAIL), armazenamento de arquivos (Google DRIVE) e editores de textos, planilhas e apresentações (Google DOCS). É possível no Google Classroom criar turmas, partilhar documentos, propor tarefas e promover discussões. De forma simples e intuitiva, o docente consegue organizar suas aulas em formato de tópicos, tendo a possibilidade de compartilhar documentos, áudios, vídeos, links, interação com outros aplicativos ou ferramentas e uma infinidade de possibilidades. Além disso, é possível criar avisos, atividades que permitem a correção, nota e feedback. O ambiente é limpo, bastando apenas o acesso via e-mail, que geralmente o estudante já possui ou pode criar dentro da plataforma Google. Sua interface é semelhante a uma rede social, onde de forma lúdica o estudante consegue visualizar todo o conteúdo de forma ágil. Há ainda a possibilidade de acesso via smartphone através do site e do aplicativo disponibilizado para Android e IOS (Araújo, 2016).

Diante deste contexto, o objetivo deste trabalho foi avaliar a percepção dos estudantes da Faculdade de Odontologia de Pernambuco (FOP/UPE) sobre o uso do aplicativo Google Classroom, como ferramenta de apoio ao ensino-aprendizagem, durante o período letivo suplementar trans pandêmico. 


\section{Metodologia}

Trata-se de uma pesquisa aplicada, descritiva, transversal, de levantamento e natureza quantitativa e qualitativa. O projeto de pesquisa foi encaminhado para o Comitê de Ética e Pesquisa, via Plataforma Brasil, mediante aprovação do sistema CEP/CONEP com parecer número 4.643.643. A pesquisa está de acordo com a resolução 466/2012-CNS/MS. Foi elaborado pelos autores um questionário com 19 itens de múltipla escolha e os resultados foram tabulados pela própria plataforma em planilhas (via Google Sheets), podendo ser visualizados em gráficos. Foi utilizado o aplicativo de formulários online: Google forms. Os dados foram analisados mediante estatística descritiva (média e desvio padrão, valor máximo e mínimo), considerando como nível de significância p-valor $\leq 0,05$ para um intervalo de confiança de $95 \%$. Os discentes regularmente matriculados no período letivo suplementar trans pandêmico na Faculdade de Odontologia de Pernambuco foram informados de todo o processo do estudo e, aqueles que aceitaram participar, assinaram o termo de consentimento livre e esclarecido (TCLE), antes de responderem o formulário. A amostra foi composta por 100 estudantes.

\section{Resultados}

A amostra foi composta por 100 alunos, do $1^{\circ}$ ao $10^{\circ}$ período (Gráfico 1). A maioria dos entrevistados foram alunos do 9 período $(22 \%)$.

Gráfico 1 - Distribuição dos 100 estudantes entrevistados de acordo com o seu período.
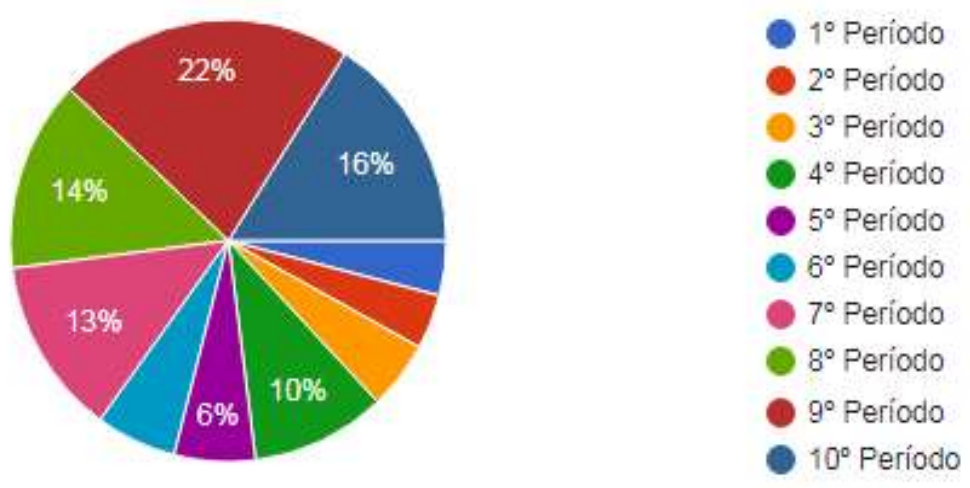

Fonte: Autores.

Dos 100 alunos entrevistados, $43 \%$ relataram demorar mais do que 1 hora na locomoção de ida e volta da faculdade (Gráfico 2). 
Gráfico 2 - Distribuição dos estudantes de acordo com as horas gastas diariamente na locomoção para ir e voltar da faculdade.

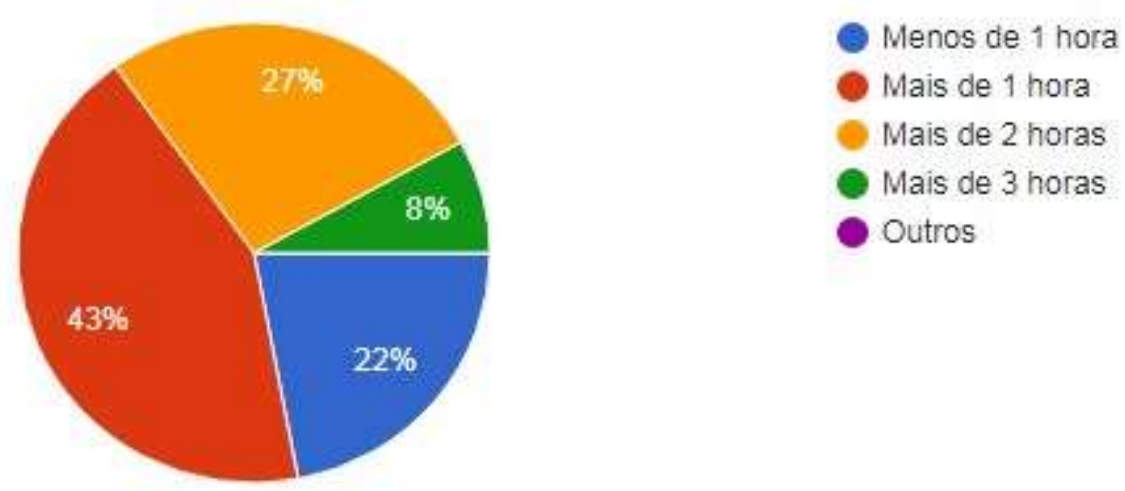

Fonte: Autores.

63\% dos participantes responderam que nunca haviam utilizado o Google Classroom como ferramenta de apoio ao ensino-aprendizagem antes do período suplementar (Gráfico 3).

Gráfico 3 - Distribuição dos estudantes de acordo com a utilização do Google Classroom como ferramenta de apoio ao ensinoaprendizagem, antes do período letivo suplementar.

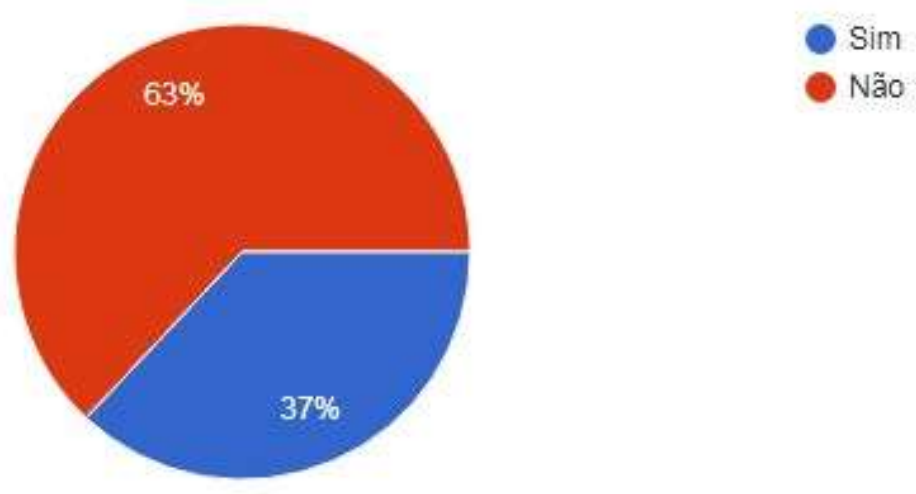

Fonte: Autores.

Em relação à quantidade de disciplinas cursadas, 30\% dos alunos cursaram cinco disciplinas (Gráfico 4), durante o ano letivo suplementar. 
Gráfico 4 - Distribuição dos estudantes de acordo com a quantidade de disciplinas cursadas durante o período letivo suplementar.
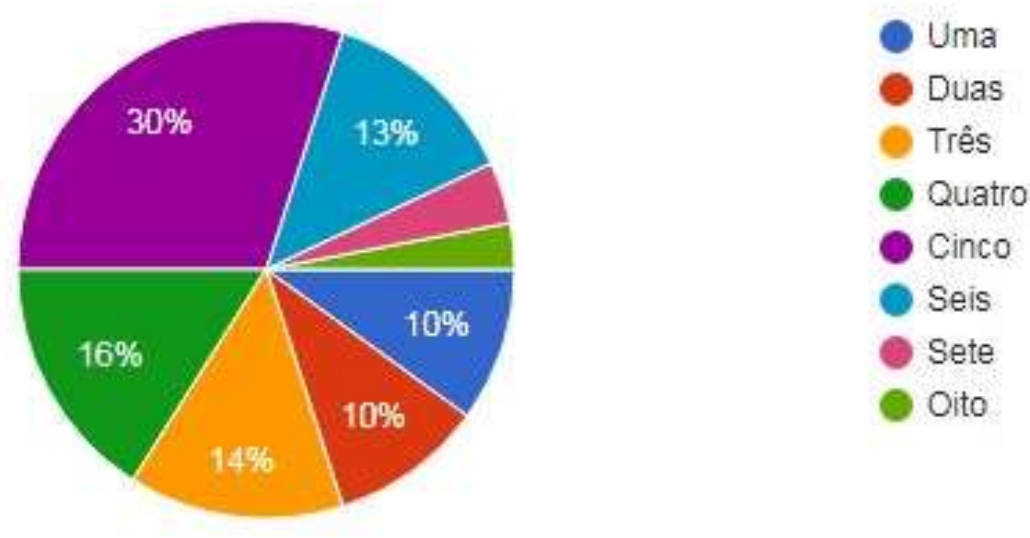

Fonte: Autores.

Quando indagados sobre qual dos dispositivos tecnológicos os alunos usavam para acessar a internet e, consequentemente o Google Classroom, $73 \%$ dos entrevistados responderam que utilizaram o notebook (Gráfico 5).

Gráfico 5 - Distribuição dos estudantes de acordo com o dispositivo utilizado para ter acesso às aulas online no Google Classroom.

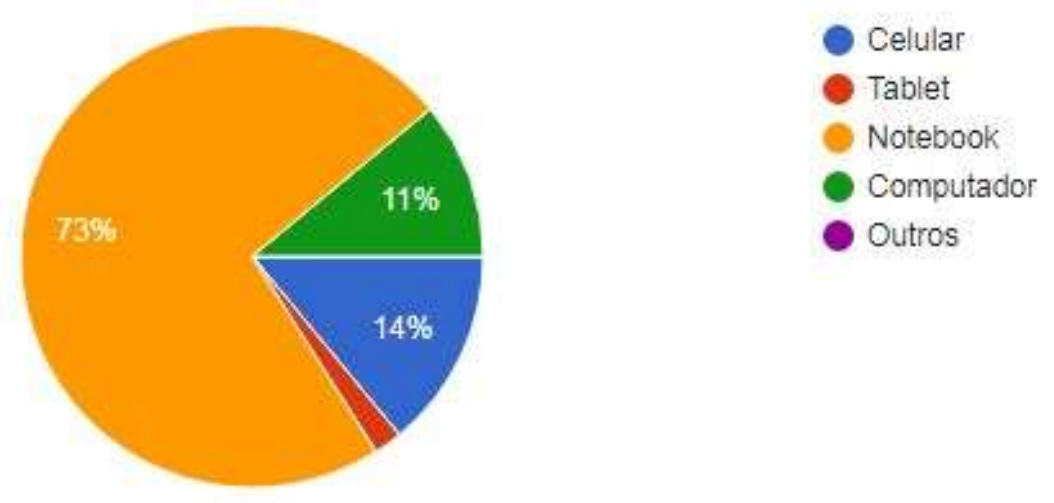

Fonte: Autores.

Quanto a utilização dos recursos do Google, durante o período letivo suplementar, $98 \%$ dos estudantes responderam que utilizaram a plataforma Google Meet (Gráfico 6). 
Research, Society and Development, v. 10, n. 15, e464101521945, 2021

(CC BY 4.0) | ISSN 2525-3409 | DOI: http://dx.doi.org/10.33448/rsd-v10i15.21945

Gráfico 6 - Distribuição dos estudantes de acordo com os recursos utilizados do Google durante o período letivo suplementar.

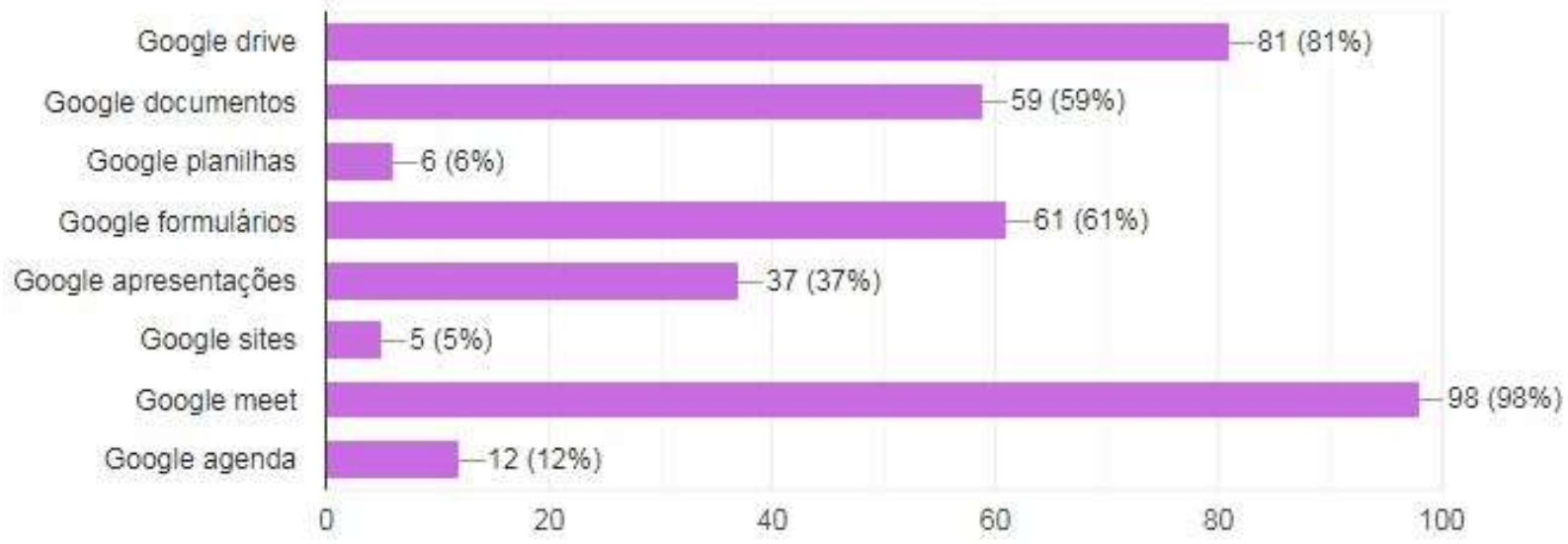

Fonte: Autores.

De acordo com às dificuldades encontradas de conexão à internet durante as aulas online, $37 \%$ dos alunos concordaram parcialmente que tiveram problemas com o acesso à internet (Gráfico 7).

Gráfico 7 - Distribuição dos estudantes de acordo com os problemas de acesso à internet durante o período letivo suplementar.

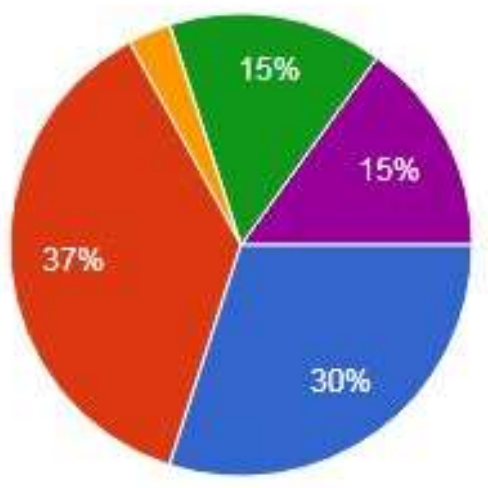

Concordo plenamente

Concordo parcialmente

Nem concordo, nem discordo

Discordo plenamente

Discordo parcialmente

Fonte: Autores.

De acordo com a facilidade de uso da plataforma Google Classroom, 59\% dos alunos concordaram plenamente que não tiveram problemas quanto ao seu uso (Gráfico 8). 
Gráfico 8 - Distribuição dos estudantes de acordo com a facilidade para utilizar o Google Classroom.

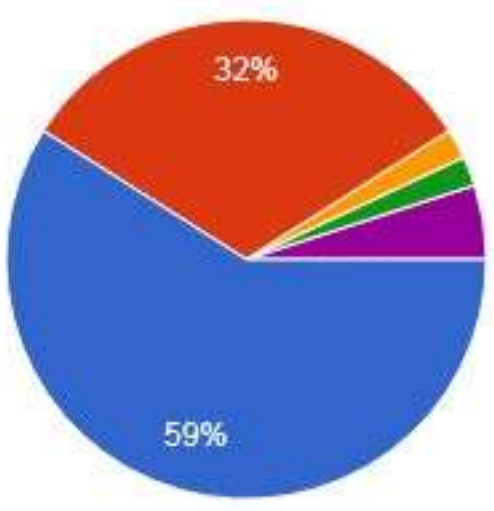

Concordo plenamente

Concordo parcialmente

Nem concordo, nem discordo

Discordo plenamente

Discordo parcialmente

Fonte: Autores.

Quando questionados se houve algum prejuízo no ensino-aprendizagem dos conteúdos utilizando o Google Classroom, a maioria dos alunos (31\%) discordam parcialmente (Gráfico 9).

Gráfico 9 - Distribuição dos estudantes de acordo com a ausência de prejuízo no ensino-aprendizagem dos conteúdos utilizando o Google Classroom.

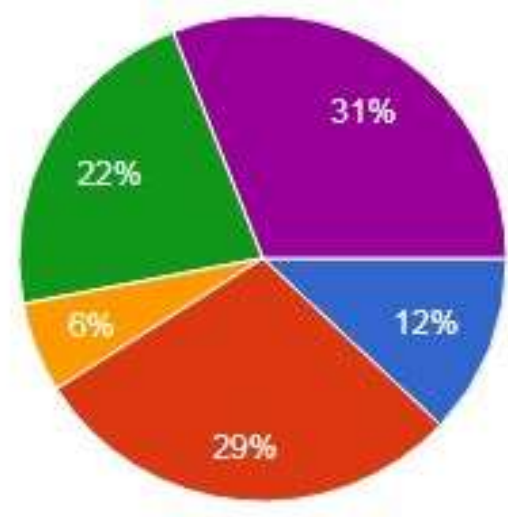

Concordo plenamente

- Concordo parcialmente

Nem concordo, nem discordo

Discordo plenamente

- Discordo parcialmente

Fonte: Autores.

Quando questionados se consideram o Google Classroom como uma ferramenta adequada para uso nas disciplinas cursadas durante o período letivo suplementar, $51 \%$ dos alunos concordaram plenamente (Gráfico 10). 
Gráfico 10 - Distribuição dos estudantes que consideram o Google Classroom uma ferramenta adequada para as disciplinas cursadas durante o período letivo suplementar.

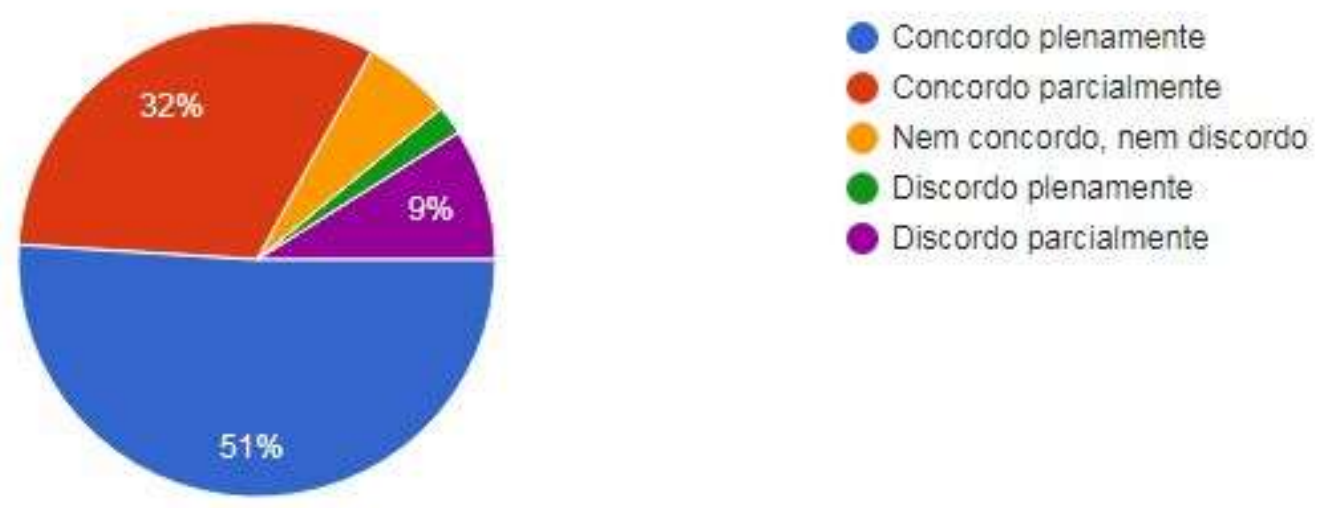

Fonte: Autores.

Em relação a capacitação dos professores quanto ao uso da ferramenta Google Classroom, como meio auxiliar de ensino, $47 \%$ dos alunos concordaram parcialmente que os professores estavam capacitados (Gráfico 11).

Gráfico 11 - Distribuição dos estudantes que consideram que os professores estavam capacitados para o uso do Google Classroom.
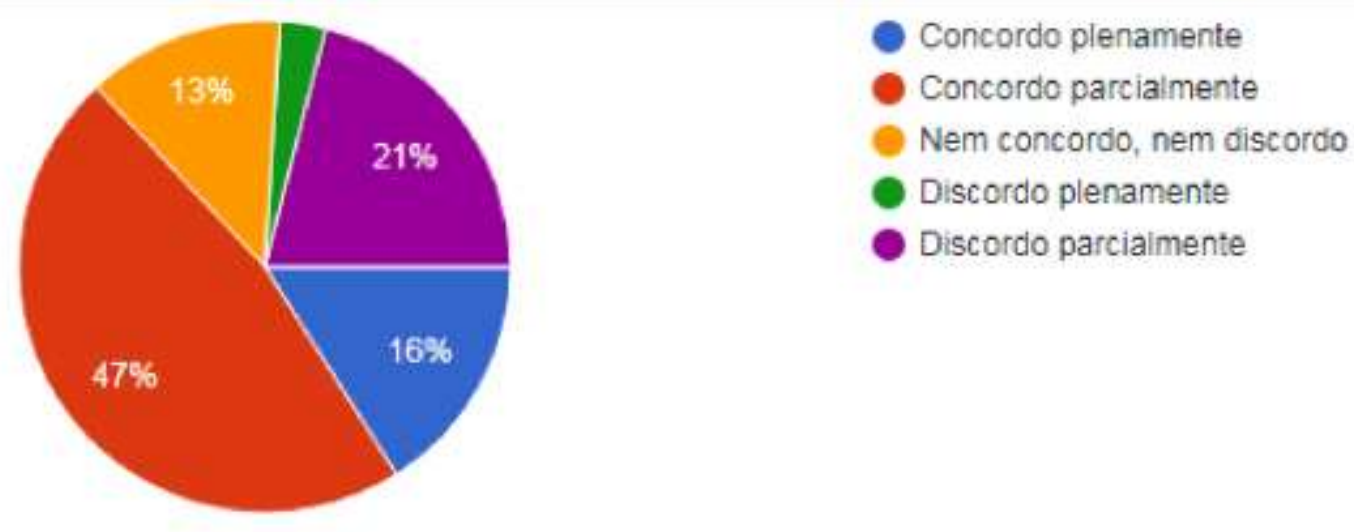

Fonte: Autores.

Quanto ao uso do Google Meet como ferramenta adequada para assistir as aulas síncronas (online) durante o período letivo suplementar, 69\% dos alunos concordaram plenamente em seu uso (Gráfico 12). 
Gráfico 12 - Distribuição dos estudantes que concordaram que o Google Meet foi uma ferramenta adequada para assistir as aulas síncronas on-line durante o período letivo suplementar.

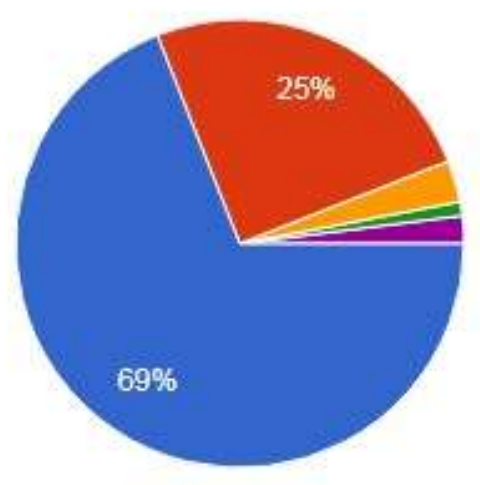

Concordo plenamente

Concordo parcialmente

Nem concordo, nem discordo

Discordo plenamente

Discordo parcialmente

Fonte: Autores.

Sobre o envio e/ou marcação e/ou adição das atividades acadêmicas propostas pelos professores, no Google Classroom, $51 \%$ dos alunos responderam apresentar facilidade na plataforma (Gráfico 13).

Gráfico 13 - Distribuição dos estudantes que concordaram que tiveram facilidade para enviar e/ou adicionar e/ou marcar como concluída as atividades acadêmicas propostas pelos professores no Google Classroom.

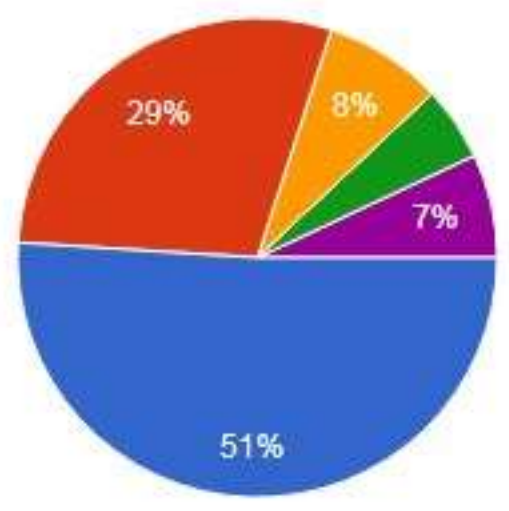

Concordo plenamente

Concordo parcialmente

Nem concordo, nem discordo

Discordo plenamente

Discordo parcialmente

Fonte: Autores.

Ao questionar sobre o acesso as notas no Google Classroom das atividades propostas pelos professores, $39 \%$ dos estudantes demonstraram facilidade na visualização (Gráfico 14). 
Gráfico 14 - Distribuição dos estudantes de acordo a facilidade para acessar a aba de "notas" do Gooogle Classroom.
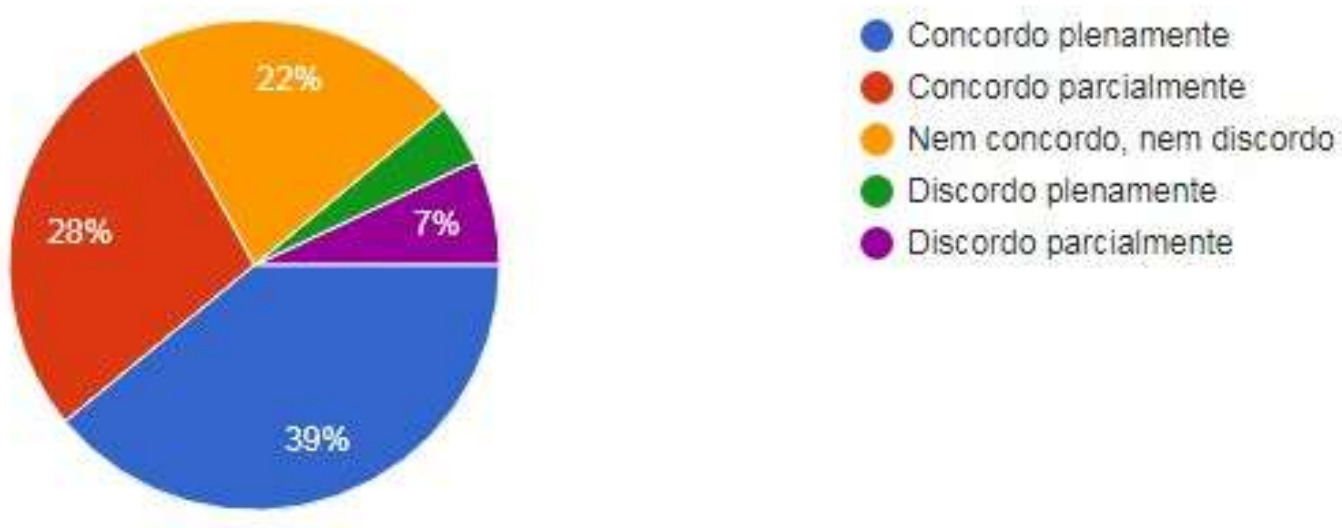

Fonte: Autores.

Quanto à facilidade para interagir com o professor e os colegas de turma, 63\% dos alunos responderam que houve facilidade na interação. (Gráfico 15).

Gráfico 15 - Distribuição dos estudantes de acordo com a facilidade de utilização da aba mural do Google Classroom para interagir com o professor e os colegas de turma.

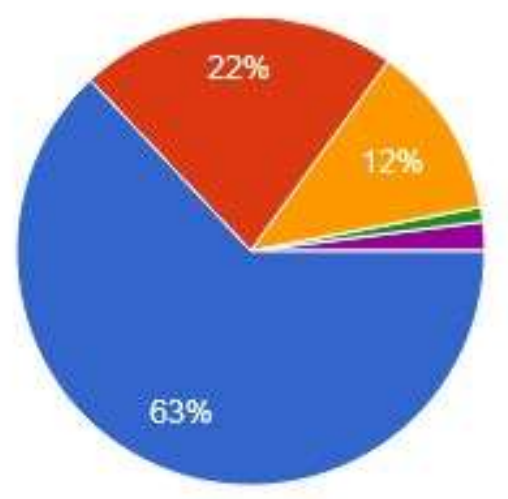

Concordo plenamente

Concordo parcialmente

Nem concordo, nem discordo

Discordo plenamente

Discordo parciaimente

Fonte: Autores.

69\% dos estudantes relataram de forma satisfatória a interação aluno/professor através da ferramenta Google Classroom (Gráfico 16). 
Gráfico 16 - Distribuição dos estudantes que concordaram que a interação aluno/professor através da ferramenta Google Classroom foi satisfatória.

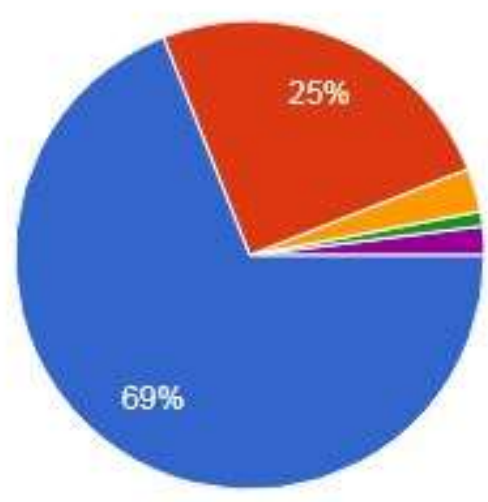

Concordo plenamente

Concordo parcialmente

Nem concordo, nem discordo

Discordo plenamente

Discordo parcialmente

Fonte: Autores.

$33 \%$ dos alunos relataram apresentar mais tempo de estudo quando da utilização da plataforma do Google Classroom (Gráfico 17).

Gráfico 17 - Distribuição dos estudantes que concordaram que tiveram mais tempo para estudar com a utilização do Google Classroom, durante o ensino on-line.

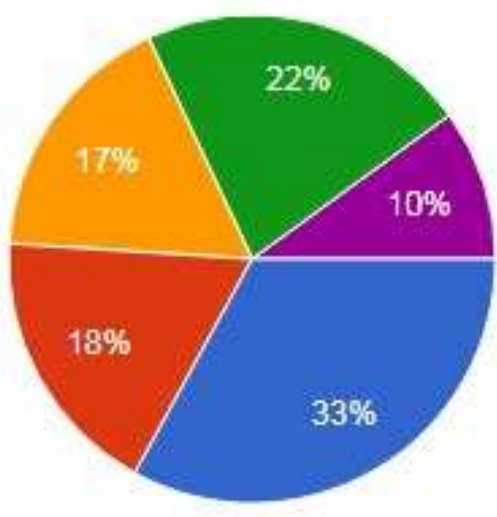

- Concordo plenamente

- Concordo parcialmente

Nem concordo, nem discordo

Discordo plenamente

- Discordo parcialmente

Fonte: Autores.

No entanto, $33 \%$ dos alunos responderam que não houve melhorias no desempenho dos estudos durante o período suplementar letivo (Gráfico 18). 
Gráfico 18 - Distribuição dos estudantes que concordam que seu desempenho nos estudos melhorou durante o período suplementar letivo.
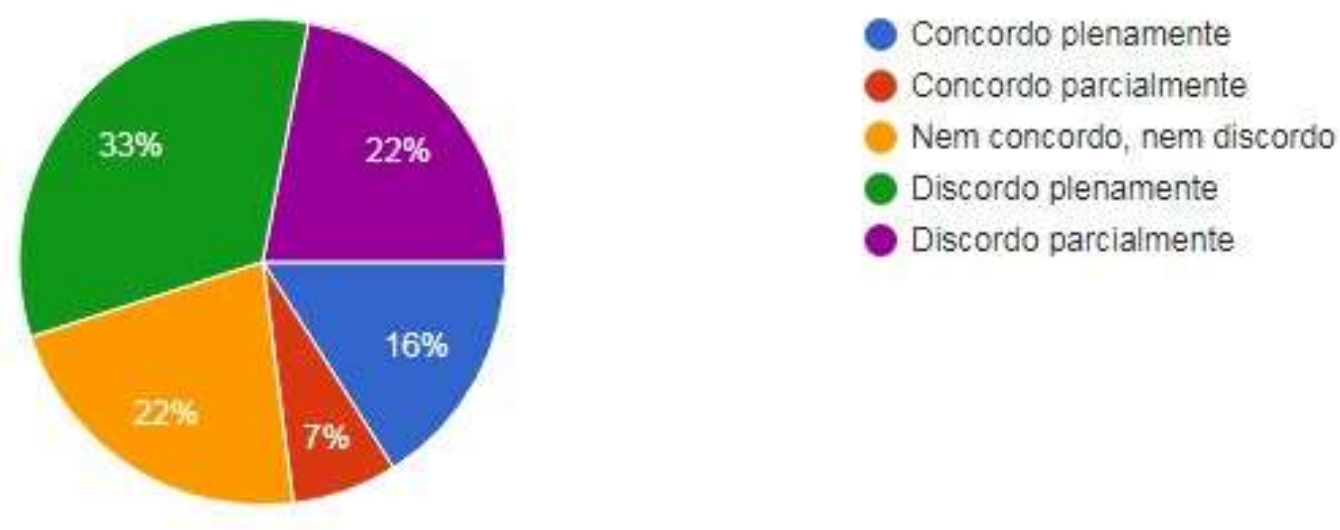

Fonte: Autores.

Quando questionados sobre a continuação do ensino online utilizando o Google Classroom para o conteúdo teórico, $39 \%$ dos alunos concordaram plenamente (Gráfico 19).

Gráfico 19 - Distribuição dos estudantes que gostariam de continuar com ensino on-line utilizando o Google Classroom para o conteúdo teórico.
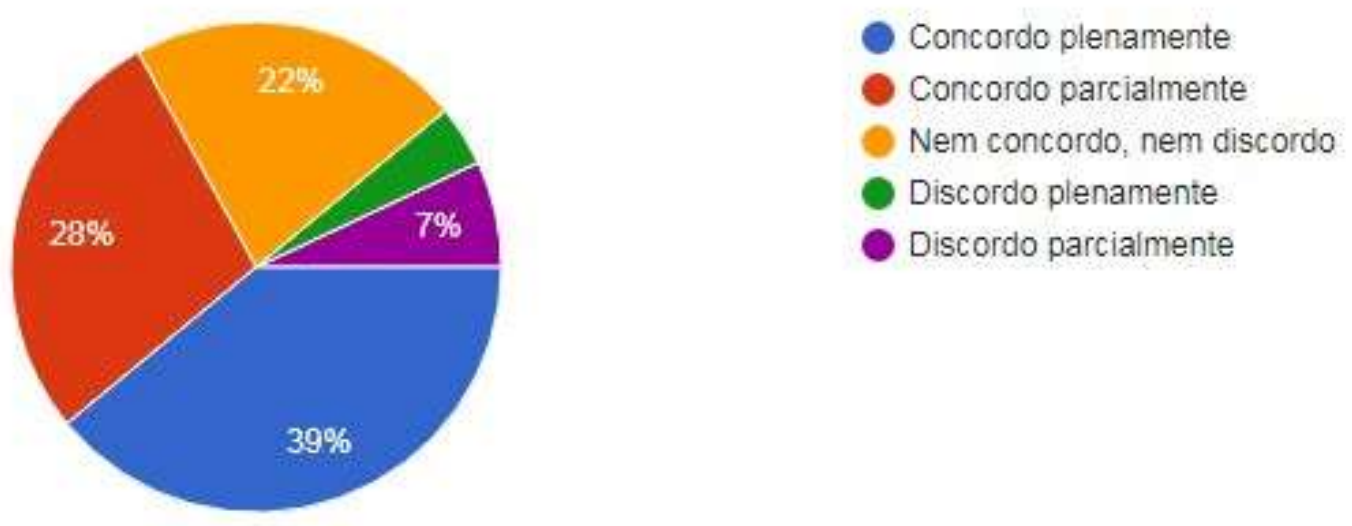

Fonte: Autores.

\section{Discussão}

A pandemia do novo coronavírus motivou a inovação das estratégias pedagógicas nos cursos de Odontologia em todo o mundo, para manter o distanciamento social e minimizar a propagação da infeção e os resultados desse estudo revelou que a implantação do Google Classroom como plataforma on-line de ensino-aprendizagem, durante o período letivo suplementar, foi percebida pelos estudantes da Faculdade de Odontologia de Pernambuco, Universidade de Pernambuco (FOP/UPE) como uma experiência positiva.

Participaram como voluntários da pesquisa, 100 estudantes matriculados em todos os semestres do curso de Odontologia (do primeiro ao décimo). A maioria informou que esta foi a primeira experiência educacional com o Google Classroom $(63 \%)$ e em média, cursaram quatro disciplinas, o que demonstra boa frequência de uso e a possibilidade de emitir opinião formada. 
Antes da pandemia as plataformas on-line de gestão de aprendizagem ou Learning Management System (LMS) disponibilizam funcionalidades para o processo de aprendizagem e possibilitavam seu gerenciamento, controle e acompanhamento, e por isso, mereciam ser exploradas pelos professores em sala de aula. (Souza \& Souza, 2016)

Em um estudo sobre a utilização do Google Classroom realizado na Universidade Federal de Alagoas, á era defendido que a educação já requisitava de uma reorganização de seus métodos e práticas, adaptando-se à sociedade cada vez mais conectada, com o uso de diferentes mídias, tornando a sala de aula mais interativa, colaborativa, com sentido real para os estudantes, promovendo a verdadeira aprendizagem significativa. (Soares, 2018)

A primeira parte do questionário eletrônico tinha como objetivo caracterizar os fatores que também poderiam influenciar no acompanhamento da utilização da plataforma educacional, como a conectividade dos estudantes, ou seja, se possuíam equipamentos adequados ou acesso à internet. A maioria respondeu que utilizaram o notebook (73\%) para ter acesso às disciplinas on-line, seguido do smartphone (14\%). O elevado número de usuários de notebook pode estar relacionado a sua popularidade, uma vez que esse dispositivo já fazia parte do cotidiano dos estudantes na prática das atividades pedagógicas e nos horários livres. Nos cursos de Odontologia, o uso das tecnologias de informação e comunicação (TIC's), como facilitadoras do conhecimento, são comprovadas e mais utilizadas em pesquisas bibliográficas, delineamentos epidemiológicos, no acesso à aplicativos móveis que orientam o diagnóstico e terapêutica dos desafios da prática clínica, além de orientações para administração e uso de marketing profissional, porém, nunca para a totalidade do ensino teórico, por se tratar de um curso presencial. (Silva et al., 2018)

$\mathrm{O}$ acesso pelo smartphone não foi tão expressivo, como ocorreu em estudos anteriores que o apontaram como o mais utilizado pelos estudantes em um curso de Biologia (Lopes, 2016) e do Bacharelado em Sistemas de Informação (Mendes \& Amorim, 2019), talvez por ser um dos equipamentos com acesso à internet mais vendido no Brasil e no mundo (Chang et al., 2020). A apropriação do estudo neste dispositivo móvel potencializa a aprendizagem móvel ou Mobile-Learning (M-Learning), uma tendência educacional que permite a utilização de recursos pedagógicos, com a premissa de que podemos aprender a qualquer hora e em qualquer lugar, implicando em colaboração e personalização do aprendizado. Alguns estudantes utilizaram computadores ou tablets, revelando uma grande vantagem do Google Classroom que permite a operacionalização, por meio de equipamentos variados e não necessita de instalação local ou um servidor dedicado (Kearney, 2012; Souza \& Souza, 2016).

Outro ponto de destaque do Google Classroom, é a conexão com o conjunto de aplicações desenvolvidas e disponibilizadas pelo Google, conhecido como G Suite for Education, permitindo sua interação com o Gmail, Google Agenda, Google Drive, Google Forms, Hangouts entre outros. Assim, amplia a gama de possibilidades que o professor tem para preparar, gerenciar e avaliar o ambiente de aprendizagem. Em relação ao uso desses aplicativos, os estudantes revelaram que o Google meet, Google drive, Google formulário, Google documentos, e Google apresentações foram os mais utilizados. O Google meet foi considerado adequado para a transmissão dos encontros síncronos pela maioria dos estudantes (51\%) e possibilitou a gravação das aulas para o acesso posterior, principalmente na ocorrência de interrupções da internet.

Apesar dos estudantes terem informado que em algum momento tiveram problemas com a instabilidade de acesso à internet (37\%), os dados supracitados demonstram que isto não foi um obstáculo para a aplicação do ensino on-line utilizando o Google Classroom, pois os estudantes possuíam os recursos necessários para a dinâmica da metodologia proposta, conseguiam acessar o conteúdo e resolver as tarefas pela plataforma.

Os resultados sobre a facilidade de utilização do Google Classroom, pelos estudantes (59\%), já eram esperados e em consonância com outros estudos na literatura, por se tratar de uma plataforma acessível, intuitiva e de simples manuseio. 
(Kearney et al., 2012; Bento \& Cavalcante, 2013; Valente, 2014; Mendes \& Amorim, 2019; Al-Marrof \& Al-Emran 2018; Bondarenk, Mantulenko \& Pikilnyak, 2019; Rahmad et al., 2019). A maioria dos estudantes também não apresentou dificuldade para enviar as tarefas propostas pelos professores das disciplinas, conferir as notas de avaliação ou feedback. Talvez, por se apresentar com uma interface semelhante às redes sociais.

Apenas $(12 \%)$ dos estudantes concordaram plenamente e $(29 \%)$ parcialmente que não houve prejuízo no ensinoaprendizagem dos conteúdos durante o período letivo suplementar, utilizando o Google Classroom. Esse resultado pode ter relação com a instabilidade da conexão, pois a maioria dos estudantes relataram que tiveram problemas com acesso à internet durante as aulas on-line. A interrupção do acesso de parcela da população às tecnologias digitais, desmotivam tanto os estudantes e professores para acompanharem as aulas on-line, porém este impasse não é exclusivo em nosso país, sendo encontrado em todo o mundo, e principalmente durante a pandemia da COVID-19, quando os acessos aumentaram exponencialmente. (Arruda, 2020)

Os estudantes concordaram totalmente $(69 \%)$ e parcialmente $(25 \%)$ que essa ferramenta era adequada para as disciplinas cursadas durante o período letivo suplementar, porém uma parcela $(27 \%)$ discorda em continuar utilizando o Google Classroom no ensino on-line e $28 \%$ discordam em continuar com as aulas teóricas pela ferramenta. O resultado surpreendeu porque os jovens de hoje estão inseridos na cibercultura, e a implementação do Google Classroom oportunizou auxliar tecnologia e educação odontológica. Talvez essa percepção tenha suporte devido ao período de instabilidade emocional, diante da maior crise sanitária vivenciada e da imprevisibilidade no futuro, ou com o déficit do próprio desempenho escolar, que a maioria (33\%) concordou não ter apresentado.

Esta percepção está na contramão do apontado por estudos que estimaram que os estudantes contemporâneos “estão sedentos por práticas menos tradicionais na sala de aula", considerando sua intimidade com as tecnologias, o que pode permitir um aprendizado mais significativo, se proporcionado como alternativa ao ensino tradicional. (Assunção \& Rodrigues, 2015) Mesmo inseridos no mundo tecnológico, a maioria dos estudantes consultados em sua pesquisa, preferíram as aulas teóricas de maneira presencial. Esse resultado talvez tenha relação com a falta de capacitação de alguns professores para ministrar a aula através dessa nova realidade, aproveitando por exemplo, seus recursos adicionais e como todo método, poderá falhar, se não houver o domínio da técnica pelo instrutor e/ou o entendimento do processo pelo estudante, para motivá-los no processo de ensino-aprendizado. (Melo Júnior, 2018)

Até mesmo o tempo para o estudo em casa, foi teoricamente maior e confirmado pela maioria dos estudantes, devido não haver necessidade de deslocamento diário para a instituição de ensino. Houve a eliminação do tempo que era gasto diariamente para esse fim, geralmente superior a uma hora, visto que os estudantes muitas vezes, eram residentes de cidades distintas na região metropolitana do Recife e a Instituição, localizada em Camaragibe. Em estudos anteriores, já correlacionava a influência do menor desempenho do estudante, com maiores distâncias percorridas, má-qualidade, e o elevado tempo gasto na locomoção. (Gomide, 2006)

Quando questionados sobre os aspectos a serem melhorados, muitos estudantes alegaram que algumas aulas se tornavam cansativas e não prendiam a atenção, e sugeriram que muitos professores não souberam utilizar todos os recursos que o aplicativo oferece, alguns apenas adaptaram o que faziam na aula presencial para o on-line, não souberam distribuir a quantidade dos conteúdos em cada encontro e alguns não dosaram o quantitativo de atividades assíncronas. Sendo assim, estratégias devem ser montadas a fim de tornar a aula interativa e prender a concentração do estudante. Deve-se levar em consideração que havia pouca experiência da maioria do corpo docente com o ensino on-line e/ou com a ferramenta e o tempo 
foi escasso para o planejamento e adequação das atividades pedagógicas, até mesmo porque, o ensino em ambiente virtual requer tempo adicional de preparação do design instrucional (Barra, 2017; Al-Marrof \& Al-Emran, 2018).

Em relação a própria performance, os estudantes referiram que em casa, a concentração e atenção foram prejudicadas, por não estarem fisicamente na sala de aula e alguns tiveram dificuldade de interagir, nos momentos síncronos, com os pares. Contudo, a tecnologia na educação pode trazer inúmeros benefícios para a modernização do processo de ensino-aprendizagem, desde que o projeto tenha embasamento pedagógico e seja utilizado de forma racional, com pleno domínio dos envolvidos (Reinaldo et al., 2016).

Deve-se também levar em consideração que toda mudança de paradigma traz dificuldades na adaptação, muitos estudantes ainda não desenvolveram o autogerenciamento que é indispensável no aprendizado remoto, a disciplina e organização dos horários para o estudo em novo ambiente, principalmente por ter sido, para muitos, a primeira experiência e por se tratar de uma instituição pública que não tinha o uso regular de ambientes virtuais de aprendizagem, se enquadrando, infelizmente, no contexto de incipiência da apropriação das tecnologias digitais no âmbito nacional. (Arruda, 2020) Diferente da maioria das instituições privadas de ensino superior. A falta de suporte técnico especializado nos casos de falhas operacionais também é um fator de influência. Estas principais barreiras existentes podem ser superadas com o aumento do investimento pelo poder público no setor, desde a compra de equipamentos tecnológicos, inovação e oferta de cursos de formação continuada aos funcionários, discentes e docentes. (Assunção \& Rodrigues, 2015)

Mesmo assim, 47\% dos estudantes concordaram parcialmente que o corpo docente estava capacitado para o uso do Google Classroom. Esta percepção pode estar fundamentada por causa da oportunidade oferecida pela coordenação do curso, do treinamento on-line mediado por um grupo de professores que já possuíam alguma experiência com a plataforma e que permitiu a vivência tanto como professor, quanto estudante de ambiente virtual, além de outros cursos disponibilizados pelo programa de extensão da Universidade. Por si só, esta plataforma cria um ambiente interativo para o compartilhamento de materiais didáticos de forma dinâmica e propicia a interação em tempo real, entre corpo docente e discente, o que deve ter contribuído para a favorável opinião dos estudantes sobre a continuidade da interação com os professores e consequentemente, influenciaram na facilitação do aprendizado. (Araújo, 2016),

No entanto, autores indicam a associação do Google Classroom às metodologias ativas, a fim de promover a construção do aprendizado com maior prazer e entretenimento (Cavalcanti \& Soares, 2009; (Dellos, 2015; Coil, Ettinger \& Eisen, 2017). A Sala de aula invertida (Flipped classroom), por exemplo, proporcionou que os estudantes realizassem estudo prévio e avaliação pré-aula, chegando no encontro síncrono com um conhecimento inicial sobre o conteúdo e com dúvidas pontuais sobre determinados assuntos, tornando as aulas mais dinâmicas e personalizadas às necessidades da turma (Just-intime Teaching). (Mendes \& Amorim, 2019)

Estudos anteriores realizados com estudantes de um curso de Enfermagem, concluíram que as metodologias ativas estimulavam o estudo constante, a independência e a responsabilidade, possibilitando a integração das dimensões biopsicossociais e preparam para o trabalho em equipe. Isso foi verificado neste estudo, quando poucos estudantes discordaram que o Google Classroom não possuía flexibilidade para as atividades pedagógicas em grupos. (Marin, 2019)

Outros estudos estimularam experiências com a ciência da gamificação, que consiste em utilizar elementos de jogos no contexto da educação, modificando assim a maneira de transmitir conhecimento e dinamizando o aprendizado dos discentes. Resultados favoráveis para utilização do aplicativo Kahoot, como um recurso de jogo digital que oferece aos professores uma oportunidade de criar questionários, pesquisas e discussões que envolvam estudantes no formato de jogo competitivo. (Dellos, 
2015) Em outro estudo foi criado um jogo de tabuleiro chamado "Gut Check: The Microbiome Game", fortalecendo o conceito do Edutainment, neologismo derivado da junção das palavras education e entertainment, conteúdos que educam enquanto entretêm. Para estes autores, os educadores devem utilizar ferramentas digitais para engajar seus estudantes em atividades significativas e divertidas, envolvendo-os na resolução de problemas, no pensamento crítico e na revisão do conhecimento do conteúdo. (Coil, Ettinger \& Eisen, 2017)

O ensino a distância (EAD) veio para ficar e será irreversível, o que já está acontecendo. Houve uma mudança cultural forçada nesses últimos 12 meses de isolamento devido à pandemia. Todas as instituições de ensino presencial estão em ensino híbrido e digital. Um projeto com educação onipresente com ensino focado no aprendizado, metodologias ativas e o desenvolvimento da autonomia do estudante para o acesso ao conteúdo, em qualquer tempo e espaço, são as melhores diretrizes a serem seguidas. Talvez, o ensino presencial puro não vai mais existir, segundo (Soares \& Antunes, 2018).

Novas investigações científicas envolvendo a comunidade acadêmica devem ser estimuladas com o objetivo de determinar outros fatores que podem influenciar na maior aceitação do Google Classroom, com coleta de dados em outros cursos da Universidade de Pernambuco e em outras instituições de ensino superior, e assim, possibilita a generalização dos resultados.

A crise também pode gerar oportunidades. O impacto da pandemia de COVID-19 afetou a educação odontológica. Toda a comunidade acadêmica deve ser flexível e disposta a enfrentar mudanças. O novo coronavírus pode forçar os educadores a revolucionar o sistema educacional. Diante disso, a partir dessa vivência, torna-se pertinente que as ferramentas e estratégias de ensino-aprendizagem da Faculdade de Odontologia de Pernambuco sejam aprimoradas para oferecer mais possibilidades de cenários e recursos ao ensino da Odontologia, de tal forma que a instituição se torne capacitada e incluída nessa nova realidade.

\section{Conclusão}

Os estudantes da Faculdade de Odontologia de Pernambuco aprovaram a usabilidade e interação do aplicativo Google Classroom, como ferramenta virtual de ensino-aprendizagem, mostrando-se adequada e eficaz, quando utilizada nas disciplinas cursadas no período letivo suplementar, na Faculdade de Odontologia de Pernambuco, da Universidade de Pernambuco, durante a pandemia da COVID-19.

\section{Referências}

Al-Maroof, R. A. S., \& Al-Emran, M. (2018). Students Acceptance of Google Classroom: An Exploratory Study using PLS-SEM Approach. International Journal of Emerging Technologies in Learning, 13(6).

Antunes, M., Barros, P. R. M., Machado, L. B., Bez, M. R., Rigo, S., \& Carvalho, M. J. S. (2018). Construção de um simulador virtual para o ensino das habilidades de pensamento crítico aos estudantes de enfermagem. Coordenação Geral, 44.

Araújo, H. M. C. (2016). O uso das ferramentas do aplicativo" Google sala de aula" no ensino de matemática.

Arruda, E. P. (2020). Educação remota emergencial: elementos para políticas públicas na educação brasileira em tempos de Covid-19. EmRede-Revista de Educação a Distância, 7(1), 257-275.

Assunção, F. D. S., \& Rodrigues, E. F. (2015). A inserção das tecnologias educacionais e reflexos no pensar-fazer dos professores e alunos no ensino fundamental. Revista Tecnologias na Educação. Ano, 7.

Barra, D. C. C., Paim, S. M. S., Sasso, G. T. M. D., \& Colla, G. W. (2018). Métodos para desenvolvimento de aplicativos móveis em saúde: revisão integrativa da literatura. Texto \& Contexto-Enfermagem, 26.

Bento, M. C. M., \& dos Santos Cavalcante, R. (2013). Tecnologias Móveis em Educação.: O uso do celular na sala de aula. Educação, cultura e comunicação, 4(7).

Coil, D. A., Ettinger, C. L., \& Eisen, J. A. (2017). Gut Check: The evolution of an educational board game. PLoS biology, 15(4), e2001984. 
Research, Society and Development, v. 10, n. 15, e464101521945, 2021

(CC BY 4.0) | ISSN 2525-3409 | DOI: http://dx.doi.org/10.33448/rsd-v10i15.21945

Dellos, R. (2015). Kahoot! A digital game resource for learning. International Journal of Instructional technology and distance learning, 12(4), 49-52.

Chang, T. Y., Hong, G., Paganelli, C., Phantumvanit, P., Chang, W. J., Shieh, Y. S., \& Hsu, M. L. (2021). Innovation of dental education during COVID-19 pandemic. Journal of Dental Sciences, 16(1), 15-20.

Gomide, A. D. Á. (2006). Mobilidade urbana, iniqüidade e políticas sociais.

de Melo Júnior, P. M. R., Maia, S. M. A. S., da Cunha, R. S. C., Júnior, E. E., \& Bollela, V. R. (2018). Sala de aula invertida para o ensino do conteúdo abertura coronária em Endodontia. Revista da ABENO, 18(2), 182-191.

Kearney, M., Schuck, S., Burden, K., \& Aubusson, P. (2012). Viewing mobile learning from a pedagogical perspective. Research in learning technology, 20(1), n1.

Lopes, L. A. (2016). Olhar digital na escola: a cibercultura nas aulas de Biologia em uma escola da periferia de Canoas, RS. Revista Tecnologia na Educação, (14), 1-12.

Marin, M. J. S., Lima, E. F. G., Paviotti, A. B., Matsuyama, D. T., Silva, L. K. D. D., Gonzalez, C., \& Ilias, M. (2010). Aspectos das fortalezas e fragilidades no uso das metodologias ativas de aprendizagem. Revista brasileira de educação médica, 34, 13-20.

Martins, J., Teles, A., Viana, D., Silva, F. J., Coutinho, L., \& Teixeira, S. (2019). Avaliação do Google Sala de Aula como Ferramenta de Apoio ao processo de ensino-aprendizagem em um curso de licenciatura em Ciências Biológicas presencial. RENOTE, 17(3), 587-596.

Reinaldo, F., Magalhães, D., Reis, L., Gaffuri, S., Freddo, A., \& Hallal, R. (2016). Uso de smartphones na educação: Avaliação por grupos focais. CIAIQ2016, 1 .

Mendes, L., \& Amorim, N. (2019, November). Uso da plataforma web Google Classroom como ferramenta de apoio à metodologia Flipped Classroom: relato de aplicação no curso de Bacharelado em Sistemas de Informação. In Anais do Workshop de Informática na Escola. 25(1), 647-656.

Silva, A. F., Pauferro, B. C. S., Cruz, G. M., Trezena, S., \& Batista, R. W. C. (2018). O uso das tecnologias de informação e comunicação no ensino e em odontologia. Rev. Academia brasileira de odontologia, 8(1), 33-39.

Soares, E. C. (2018). O google sala de aula como interface de aprendizagem no ensino superior. Simpósio Internacional de Educação e ComunicaçãoSIMEDUC, (9).

Souza, A. C. S. D. (2016). Uso da Plataforma Google Classroom como ferramenta de apoio ao processo de ensino e aprendizagem: Relato de aplicação no ensino médio.

Valente, J. A. (2014). A comunicação e a educação baseada no uso das tecnologias digitais de informação e comunicação. UNIFESO-Humanas $e$ Sociais, $1(01), 141-166$. 